\title{
The clinical features of foreign body aspiration into the lower airway in geriatric patients
}

This article was published in the following Dove Press journal:

Clinical Interventions in Aging

24 September 2014

Number of times this article has been viewed

\author{
Lianjun Lin' \\ Liping Lv'* \\ Yuchuan Wang' \\ Xiankui Zha ${ }^{2}$ \\ Fei Tang ${ }^{2}$ \\ Xinmin Liu',* \\ 'Geriatric Department, Peking \\ University First Hospital, Beijing, \\ People's Republic of China; \\ 2Pulmonary Intervention Department, \\ Anhui Chest Hospital, Hefei, People's \\ Republic of China \\ *These authors contributed equally \\ to this work
}

Correspondence: Lv Liping Pulmonary Intervention Department, Anhui Chest Hospital, Ji Xi Street No 397, Dao Xiangcun Avenue, Shushan District, Hefei 230022, Anhui Province, People's Republic of China

Tel +8655I 63636374

Email Ivliping1759@sina.com

Liu Xinmin

Geriatric Department, Peking University First Hospital, Xishiku Avenue 8, Xicheng District, Beijing 100034, People's

Republic of China

Tel +86 I0 83572I 28

Email Ixm2128@163.com
Purpose: To analyze the clinical features of foreign-body aspiration into the lower airway in geriatric patients.

Patients and methods: The clinical data of 17 geriatric patients with foreign-body aspiration were retrospectively analyzed and compared with 26 nongeriatric adult patients. The data were collected from Peking University First Hospital and Anhui Chest Hospital between January 2000 and June 2014.

Results: (1) In the geriatric group, the most common symptoms were cough and sputum (15 cases, 88\%), dyspnea (six cases, 35\%), and hemoptysis (four cases, 24\%). Five patients $(29 \%)$ in the geriatric group could supply the history of aspiration on their first visit to doctor, a smaller percentage than in the nongeriatric group (13 cases, 50\%). Only three cases in the geriatric group were diagnosed definitely without delay. Another 14 cases were misdiagnosed as pneumonia or lung cancer, and the time of delayed diagnosis ranged from 1 month to 3 years. Complications due to delay in diagnosis included obstructive pneumonitis, atelectasis, lung abscess, and pleural effusion. (2) Chest computed tomography demonstrated the foreign body in three cases $(21 \%)$ in the geriatric group, which was lower than the positive proportion of detection in the nongeriatric group (nine cases, 35\%). The most common type of foreign body in the geriatric group was food, such as bone fragments (seven cases, $41 \%$ ) and plants (seven cases, $41 \%$ ), and the foreign body was most often lodged in the right bronchus tree (eleven cases, 65\%), especially the right lower bronchus (seven cases, 41\%). Flexible bronchoscopy removed the foreign body successfully in all patients.

Conclusion: The clinical features of foreign-body aspiration in geriatric patients are usually more obscure than in nongeriatric adults, which may lead to long delay in diagnosis. Flexible bronchoscopy is safe and useful for early diagnosis and effective management in geriatric patients. We suggest flexible bronchoscopy as the first-line approach to similar patients, especially those with aspiration history and unexplained pneumonia.

Keywords: airway foreign-body aspiration, flexible bronchoscopy, geriatric patients

\section{Introduction}

Tracheobronchial airway foreign-body aspiration may be a serious medical problem associated with significant morbidity and mortality. Although foreign-body aspiration into the lower airway is less common in adults than in children, the incidence in and damage to adults cannot be ignored. ${ }^{1}$ Especially for geriatric patients, its incidence increases with aging, and aspiration can be a life-threatening condition. The prevalence of airway foreign-body aspiration increases steadily with age, beginning in the sixth decade. ${ }^{1}$

However, the clinical presentation may be more obscure in elderly patients than in other adults, probably due to uncharacteristic symptoms, unclear history of foreign 
body aspiration, false-negative results of direct presentation by chest computed tomography (CT), delayed application of flexible bronchoscopy, and other reasons. Identification and removal of the foreign body is of vital importance to avoid complications such as recurrent pneumonia, hemoptysis, and granulation formation. However, the information about airway foreign-body aspiration in geriatric patients is limited. To investigate the clinical features of airway foreign-body aspiration in geriatric patients and to improve diagnosis and treatment in this patient group, we analyzed the clinical data of 17 geriatric patients with foreign-body aspiration over the past 14 years and compared them with results from 26 nongeriatric adult patients in the same period.

\section{Materials and methods Study design}

In this retrospective study, we analyzed the medical records of adults patients with foreign-body aspiration treated at Peking University First Hospital or Anhui Chest Hospital between January 2000 and June 2014. Patients older than 18 years of age with definitively diagnosed foreign-body aspiration into the lower airway were enrolled. Symptoms, aspiration history, chest CT presentations, and treatments were reviewed. The nature and lodgment site of the foreign body were recorded. Patients older than 65 years of age were included in the geriatric group.

\section{Endoscopic procedures}

Flexible bronchoscopes were used in all patients. Topical anesthesia was given intranasally using lidocaine for the first attempt of flexible bronchoscopy in all the patients. Patients with a high risk for hemorrhage and asphyxia (eight patients) were given general anesthesia for the second attempt of flexible bronchoscopy. The accessories to remove the foreign bodies included foreign-body forceps, biopsy forceps, snares, and foreign-body baskets. Other techniques included argon plasma coagulation and cryotherapy.

\section{Data analysis}

The data of the patients, including age, sex, symptoms, chest CT images, process of diagnosis and treatment, and endoscopic findings, including the types and locations of foreign body, were collected and analyzed.

\section{Results \\ Clinical manifestations}

Of the 43 adults examined, 30 were male and 13 were female. The range of age was from 18-83 years, with a mean age of 56 years. Seventeen patients ( $40 \%$ ) were older than 65 years of age. The most common symptoms among these patients were cough and sputum (15 cases, 88\%), dyspnea (six cases, 35\%), and hemoptysis (four cases, 24\%) (Table 1). Although five of the 17 patients ( $30 \%$ ) could supply the history of aspiration on their first visit to doctor, only three cases were immediately definitively diagnosed. The remaining 14 cases were misdiagnosed as pneumonia (13 cases) or lung cancer (one case), and the time of delay in diagnosis ranged from 1 month to 3 years. In the nongeriatric group $(n=26), 13$ patients $(50 \%)$ could supply the history of aspiration on their first visit to doctor. Two patients were immediately definitively diagnosed. The remaining 24 cases were misdiagnosed as pneumonia (23 cases) or lung cancer (one case) and the time of delayed diagnosis ranged from 6 days to 10 years.

\section{Chest CT}

The most common presentation of foreign-body aspiration on chest CT was pneumonic patch (13 cases, 93\%). In the geriatric group, chest CT identified the foreign body in three patients $(21 \%)$, while in the nongeriatric group, it was detectable in nine patients $(35 \%)$. The nondirect presentation on chest CT findings is shown in Table 2.

\section{Foreign body}

The types of foreign bodies are listed in Table 3. Food accounted for more than $80 \%$ of foreign-body features, both

Table I Characteristics of symptoms and aspiration history of patients

\begin{tabular}{lll}
\hline & $>\mathbf{6 5}$ years, cases (\%) & $\leq \mathbf{6 5}$ years, cases $(\%)$ \\
\hline Cough, sputum & $15(88)$ & $25(96)$ \\
Dyspnea & $6(35)$ & $6(23)$ \\
Hemoptysis & $4(24)$ & $1(4)$ \\
Fever & $2(12)$ & $2(8)$ \\
Chest pain & $1(6)$ & $4(15)$ \\
Supply the history of aspiration initially & $5(29)$ & $13(50)$ \\
Recall the history of aspiration after flexible bronchoscopy & $9(53)$ & $12(46)$ \\
Time for correct diagnosis & $0-3$ years & $0-10$ years \\
\hline
\end{tabular}


Table 2 Chest CT findings

\begin{tabular}{|c|c|c|}
\hline CT & $>65$ years, cases $(\%)$ & $\leq 65$ years, cases $(\%)$ \\
\hline 40 cases & I 4 cases & 26 cases \\
\hline Pneumonic patch & $13(93)$ & $24(92)$ \\
\hline Foreign body & $3(2 I)$ & $9(35)$ \\
\hline Atelectasis & $2(14)$ & $6(23)$ \\
\hline High density lesion in airway & $4(29)$ & I (4) \\
\hline Pleural effusion & 0 & $2(8)$ \\
\hline Occupy lesion & I (7) & I (4) \\
\hline Lung abscess & 0 & I (4) \\
\hline Mediastinal emphysema & 0 & I (4) \\
\hline Tracheoesophageal fistula & 0 & I (4) \\
\hline
\end{tabular}

Abbreviation: CT, computed tomography.

in the geriatric group and the nongeriatric group. In the geriatric group, bones, including five cases of chicken bones and two cases of fish bones, accounted for $41 \%$ of foreign bodies (Figure 1), similar to the percentage $(50 \%)$ in the nongeriatric group. Plants and melon seeds were easily seen, with a proportion of $41 \%$ in the geriatric group (Figure 2). The proportion of iatrogenic foreign bodies (such as dentures and trachea cannulas after tracheal incision) was much higher in the geriatric group. The location and frequency of foreignbody aspiration is shown in Table 4. Lodgment was more frequently observed in the right bronchial tree.

\section{Flexible bronchoscopy}

In the geriatric group, 14 patients (79\%) were misdiagnosed as having pneumonia or suspicion of lung cancer; the misdiagnoses persisted for 1 month to 3 years. In the nongeriatric group, more than $90 \%$ of patients were misdiagnosed as having other respiratory disease, also for several years. All patients were confirmed as having experienced foreign-body aspiration by flexible bronchoscopy. All of the foreign bodies were successfully removed by flexible bronchoscopy. In the geriatric group, 13 cases (76\%) were resolved successfully on the first attempt and another four cases (24\%) were resolved successfully on the second attempt. In the nongeriatric group, the success rate of the first attempt to remove the foreign body by flexible bronchoscopy was $85 \%$ ( 22 cases); four patients $(15 \%)$ required a second flexible bronchoscopy. Among the

Table 3 Types of foreign body

\begin{tabular}{lll}
\hline & $\begin{array}{l}>65 \text { years, } \\
\text { cases (\%) }\end{array}$ & $\begin{array}{l}\leq 65 \text { years, } \\
\text { cases }(\%)\end{array}$ \\
\hline Bones & $7(4 I)$ & $13(50)$ \\
Vegetable/melon seeds & $7(4 I)$ & $9(35)$ \\
Metal & 0 & $1(4)$ \\
Plastic & 0 & $2(8)$ \\
latrogenic & $3(18)$ & $1(4)$ \\
\hline
\end{tabular}

foreign bodies, the longest fish bone was almost $5 \mathrm{~cm}$ long, and it was cut into two segments and removed successfully by flexible bronchoscopy (Figure 1). No management-related complications were observed.

\section{Discussion}

Airway foreign-body aspiration occurs more commonly in children, and about $75 \%$ of cases occur in children younger than 3 years of age. .,3 $^{2}$ Although foreign-body aspiration is relatively rare for adults, when it does occur it is usually seen in adults older than 60 years of age, ${ }^{3,4}$ which is probably due to abnormal airway protection mechanisms. Aging-associated pathological status, impaired cough reflex and dysphagia, medications, and many other circumstances may contribute to
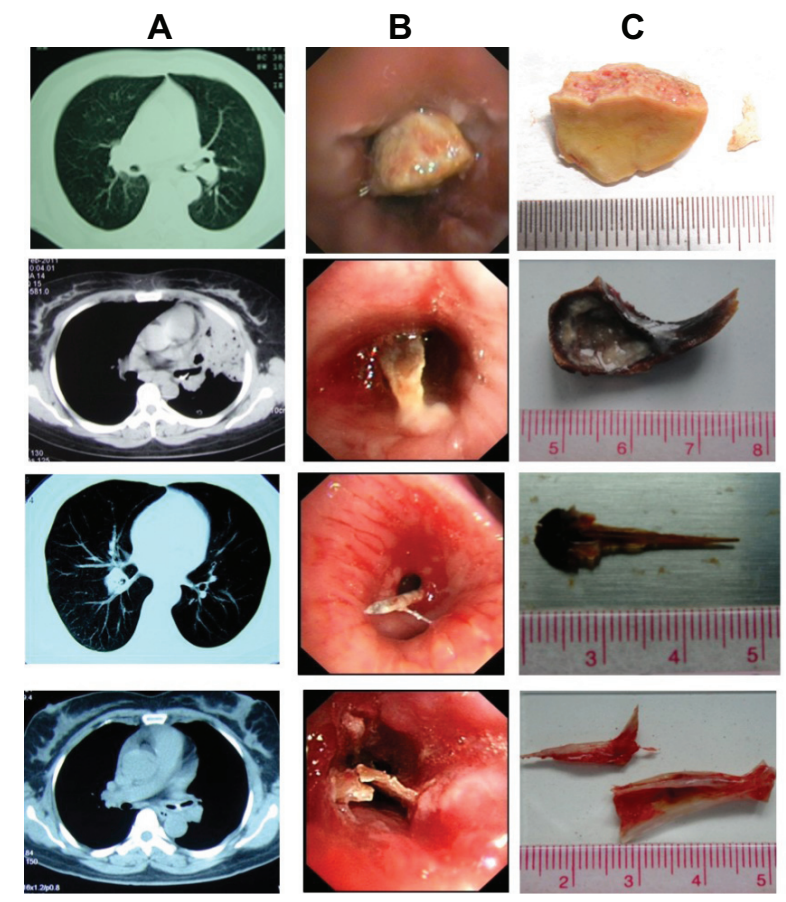

Figure I The presentation of airway foreign bodies (chicken bones and fish bones). Notes: Chest computed tomography (A), bronchoscopy (B), and photographs of the foreign bodies (C). 
A
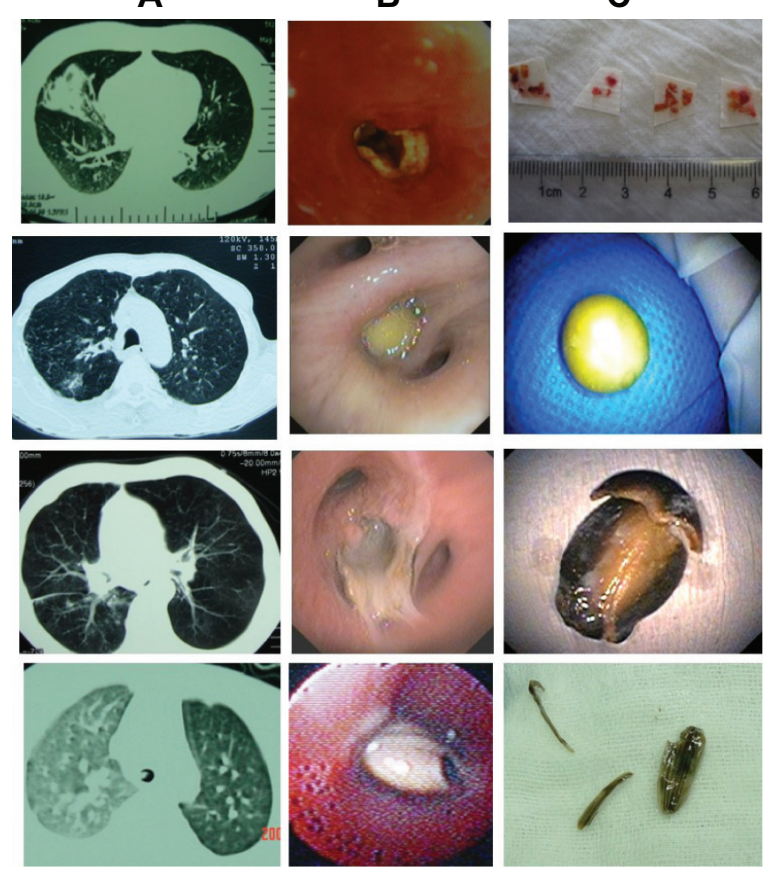

Figure 2 The presentation of airway foreign bodies (chili, pea, melon seed, and sunflower seed).

Notes: Chest computed tomography (A), bronchoscopy (B), and photographs of the foreign bodies (C).

the increased risk for airway foreign-body aspiration events in the elderly. However, the clinical information about geriatric airway foreign-body aspiration is limited. To understand the clinical features of airway foreign-body aspiration in geriatric patients for better diagnosis and treatment, we retrospectively analyzed the data of geriatric patients.

Because of the nonspecificity of clinical presentations, adult airway foreign-body aspiration is easily misdiagnosed, especially for older patients. ${ }^{4}$ Diagnosis can be delayed for months to years, and delayed diagnosis may lead to complications and potential morbidity. ${ }^{3,5}$ In our study, we found that the clinical features of airway foreign-body aspiration were usually more obscure in geriatric patients than in nongeriatric adults, which may be one of the reasons for delay in diagnosis. The nonspecificity of symptoms, the absence

Table 4 The location and frequency of foreign-body aspiration

\begin{tabular}{lll}
\hline & $\begin{array}{l}>5 \text { years, } \\
\text { cases (\%) }\end{array}$ & $\begin{array}{l}\leq \mathbf{6 5} \text { years, } \\
\text { cases (\%) }\end{array}$ \\
\hline Left & $6(35)$ & $8(3 \mathrm{I})$ \\
Left main stem & $5(29)$ & $5(19)$ \\
Left lower lobe & $\mathrm{I}(6)$ & $3(12)$ \\
Right & $\mathrm{II}(65)$ & $\mathrm{I} 8(69)$ \\
Right main stem & $\mathrm{I}(6)$ & $\mathrm{I}(4)$ \\
Right bronchus intermedius & $2(12)$ & $7(27)$ \\
Right middle lobe & $\mathrm{I}(6)$ & 0 \\
Right lower lobe & $7(4 \mathrm{I})$ & $10(38)$ \\
\hline
\end{tabular}

of or ignorance of aspiration history, and the lack of direct evidence of the existence of a foreign body on chest CT were the probable reasons for misdiagnosis in geriatric patients. We will discuss these in detail below.

As far as the symptoms are concerned, the clinical presentation of lower airway foreign-body aspiration is dependent on the site of foreign-body impaction. If the foreign body is aspirated into the tracheal region, inspiratory stridor with bouts of coughing can occur. If the foreign body passes into the bronchi, the symptoms can be variable and can range from minimal to obvious, such as coughing, sputum, wheezing, and choking. But the classic triad of cough, wheezing, and choking is only observed in a small percentage of patients. ${ }^{4,6}$ In our study, the common symptoms in the geriatric group were cough, sputum, dyspnea, and hemoptysis, similar to the results in published literature. ${ }^{4}$ No classic triad was observed. These symptoms may be obscure and nonspecific and may mimic other respiratory diseases, such as pneumonia or bronchitis, or be regarded as the exacerbation of basal disease in geriatric patients (for example, acute exacerbation of chronic obstructive pulmonary disease). Nonspecific respiratory symptoms may be mistakenly attributed to other medical conditions unless there is a clear history of aspiration, with enough attention paid to this.

Due to the little contribution of symptoms to the confirmative diagnosis of airway aspiration, doctors should emphasize the importance of aspiration history. In our study, about $30 \%$ of geriatric patients and $50 \%$ of nongeriatric patients could supply their history of aspiration on their first visit to doctor. The low proportion of recognition of aspiration in the geriatric group was probably due to the confused or damaged mental status associated with aging or disease. For example, an elderly person with neurological impairment may not be able to provide a history sufficient to suggest foreign-body aspiration. In our study, about $50 \%$ patients could recall the history of aspiration after confirmative diagnosis and treatment with flexible bronchoscopy. If doctors had been alert enough to foreign-body aspiration in adults, the aspiration history should have been collected through careful inquiry. If the aspiration history was collected and enough attention to it was paid, the time of delayed diagnosis would not have been so long and the complications could probably have been avoided.

If the aspiration history is not suggestive, chest CT may assist in detecting the foreign body. Chest CT has been demonstrated to detect unsuspected aspirated foreign bodies. ${ }^{7}$ In our study, chest CT demonstrated a foreign body in $21 \%$ of geriatric patients, which was lower than the proportion 
positively demonstrated in the nongeriatric group (35\%). The most reliable CT finding for airway foreign-body aspiration should be demonstration of the foreign body in the lumen of the tracheobronchial tree. Other indirect and nonspecific findings include the following: pneumonic patch, atelectasis, bronchiectasis, lobar consolidation, treein-bud opacities, ipsilateral pleural effusion, ipsilateral hilar lymphadenopathy, thickened bronchial wall next to a foreign body, and so on. ${ }^{7}$ The negative results of direct existence of a foreign body by chest CT should never exclude the possibility of airway foreign-body aspiration. Limitations of $\mathrm{CT}$ are largely dependent on the physical properties of the aspirated material and the slice thickness relative to the size of the foreign body. Radiopaque materials, such as metal or bone, may be easily detected on chest CT. If the foreign body is plant or plastic, it is not likely to be detected directly by chest $\mathrm{CT}$. Thus, a negative chest $\mathrm{CT}$ should never obviate the existence of the foreign body and the need for direct visualization of the airways.

Untreated or unrecognized foreign-body aspiration can result in obstructive pneumonitis, atelectasis and lung abscess, pneumomediastinum, or pneumothorax. ${ }^{4,8}$ Flexible bronchoscopy is effective both in the diagnosis and removal of foreign bodies. In our study, $80 \%-90 \%$ of patients were misdiagnosed as having pneumonia or suspicion of lung cancer for months to years, until flexible bronchoscopy properly confirmed the presence of a foreign body. The longest foreign body $(5 \mathrm{~cm})$ was removed successfully and skillfully by flexible bronchoscopy. All of the foreign bodies were successfully removed by flexible bronchoscopy, without complication. Our study showed that the application of flexible bronchoscopes was safe, effective, and valuable for the geriatric patients.

It has been 117 years since Gustav Killian performed the first bronchoscopy in 1897 to treat a patient with foreign-body aspiration by esophagoscope. ${ }^{9}$ Since then, bronchoscopy has become a common practice in the evaluation, diagnosis, and treatment of foreign-body aspiration. With the development of bronchoscopy, the mortality rate associated with foreignbody aspiration has decreased from $50 \%$ to less than $1 \%{ }^{10,11}$ But as far as geriatric patients are concerned, the application of flexible bronchoscopy is not so widely used as it is in nongeriatric adult patients, probably because of complicated basal disease or weak cardiac and pulmonary function. Due to the high misdiagnosis rate in geriatric patients, flexible bronchoscopy should be performed actively in all cases of suspected foreign-body aspiration, even if the aspiration history and radiological findings are normal. ${ }^{12,13}$ Airway foreign bodies can be removed safely and effectively with flexible bronchoscopy by experienced experts.

\section{Conclusion}

Foreign-body aspiration presents more commonly with increasing age. The symptoms are not specific for aspiration in geriatric people. Aspiration history is essential for a diagnosis but may be obscure in older patients. However, even without a supported history of foreign-body aspiration, a high index of suspicion should be maintained for all adults, especially patients with advanced age. Chest CT may be helpful, but negative radiological results as to the existence of a foreign body cannot exclude the diagnosis of foreign-body aspiration. Flexible bronchoscopy has been demonstrated to be both safe and effective in the diagnosis and treatment of foreign-body aspiration in geriatric patients. Therefore, flexible bronchoscopy should be considered as the first-line approach to both confirm suspected cases of tracheobronchial foreign-body aspiration and to facilitate foreign-body removal as early as possible.

\section{Acknowledgment}

This work was supported by the National Natural Science Foundation of China (NSFC) (No: 81141001, 81270114).

\section{Disclosure}

The authors report no conflicts of interest in this work.

\section{References}

1. Boyd M, Chatterjee A, Chiles C, Chin R Jr. Tracheobronchial foreign body aspiration in adults. South Med J. 2009;102(2):171-174.

2. Hsu WC, Sheen TS, Lin CD, Tan CT, Yeh TH, Lee SY. Clinical experiences of removing foreign bodies in the airway and esophagus with a rigid endoscope: a series of 3,217 cases from 1970 to 1996. Otolaryngol Head Neck Surg. 2000;122(3):450-454.

3. Baharloo F, Veyckemans F, Francis C, Biettlot MP, Rodenstein DO. Tracheobronchial foreign bodies: presentation and management in children and adults. Chest. 1999;115(5):1357-1362.

4. Chen CH, Lai CL, Tsai TT, Lee YC, Perng RP. Foreign body aspiration into the lower airway in Chinese adults. Chest. 1997;112(1): 129-133.

5. al-Majed SA, Ashour M, al-Mobeireek AF, al-Hajjaj MS, Alzeer AH, al-Kattan K. Overlooked inhaled foreign bodies: late sequelae and the likelihood of recovery. Respir Med. 1997;91(5):293-296.

6. Banerjee A, Rao KS, Khanna SK, et al. Laryngo-tracheo-bronchial foreign bodies in children. J Laryngol Otol. 1988;102(11):1029-1032.

7. Zissin R, Shapiro-Feinberg M, Rozenman J, Apter S, Smorjik J, Hertz M. CT findings of the chest in adults with aspirated foreign bodies. Eur Radiol. 2001;11(4):606-611.

8. Williams GS, Modi C, Singh T, Bhabra M. A case of black magic. South Med J. 2008;101(5):552-553.

9. Zöllner F. Gustav Killian, father of bronchoscopy. Arch Otolaryngol. 1965;82(6):656-659.

10. Aytaç A, Yurdakul Y, Ikizler C, Olga R, Saylam A. Inhalation of foreign bodies in children. Report of 500 cases. J Thorac Cardiovasc Surg. 1977;74(1):145-151. 
11. de Sousa ST, Ribeiro VS, de Menezes Filho JM, dos Santos AM, Barbieri MA, de Figueiredo Neto JA. Foreign body aspiration in children and adolescents: experience of a Brazilian referral center. $J$ Bras Pneumol. 2009;35(7):653-659.

12. Orji FT, Akpeh JO. Tracheobronchial foreign body aspiration in children: how reliable are clinical and radiological signs in the diagnosis? Clin Otolaryngol. 2010;35(6):479-485.
13. Korlacki W, Korecka K, Dzielicki J. Foreign body aspiration in children: diagnostic and therapeutic role of bronchoscopy. Pediatr Surg Int. 2011;27(8):833-837.

\section{Publish your work in this journal}

Clinical Interventions in Aging is an international, peer-reviewed journal focusing on evidence-based reports on the value or lack thereof of treatments intended to prevent or delay the onset of maladaptive correlates of aging in human beings. This journal is indexed on PubMed Central, MedLine,
CAS, Scopus and the Elsevier Bibliographic databases. The manuscript management system is completely online and includes a very quick and fair peer-review system, which is all easy to use. Visit http://www.dovepress. com/testimonials.php to read real quotes from published authors. 